\title{
HOMOGENIZATION ON ARBITRARY MANIFOLDS
}

\author{
GONZALO CONTRERAS, RENATO ITURRIAGA, AND ANTONIO SICONOLFI
}

\begin{abstract}
We describe a setting for homogenization of convex hamiltonians on abelian covers of any compact manifold. In this context we also provide a simple variational proof of standard homogenization results.
\end{abstract}

\section{INTRODUCTION}

In this paper we propose a setting in which homogenization results for the HamiltonJacobi equation which have only been obtained on the torus $\mathbb{T}^{n}=\mathbb{R}^{n} / \mathbb{Z}^{n}$, or equivalently in $\mathbb{R}^{n}$ with $\mathbb{Z}^{n}$-periodic conditions, can be carried out on arbitrary compact manifolds in a natural way. Moreover we show a simple proof of the homogenization result.

A homogenization result refers to the convergence of solutions $u_{\epsilon}$ of a problem $P_{\epsilon}$ with an increasingly fast variation, parametrized by $\epsilon$, to a function $u_{0}$, solution of an "averaged" problem $P_{0}$.

We choose to present the simplest model of homogenization introduced in the celebrated paper by Lions, Papanicolaou and Varadhan [8], leaving more sophisticated versions for future work. We believe that this setting will allow to translate many classical homogenization results in $\mathbb{T}^{n}$ to more general manifolds.

A Tonelli Hamiltonian on the torus $\mathbb{T}^{n}=\mathbb{R}^{n} / \mathbb{Z}^{n}$ is a $C^{2}$ function $H: \mathbb{R}^{n} \times \mathbb{R}^{n} \rightarrow \mathbb{R}$ which is

(a) $\mathbb{Z}^{n}$-periodic, i.e. $H(x+\mathbf{z}, p)=H(x, p)$, for all $\mathbf{z} \in \mathbb{Z}^{n}$.

(b) Convex: The Hessian $\frac{\partial^{2} H}{\partial p^{2}}(x, p)$ is positive definite for all $(x, p)$.

(c) Superlinear: $\lim _{p \rightarrow \infty} \frac{H(x, p)}{|p|}=+\infty$, uniformly on $x$.

In the setting of [8] one considers a small parameter $\varepsilon>0$ and the initial value problem for the Hamilton-Jacobi equation

$$
\begin{gathered}
\partial_{t} u^{\varepsilon}+H\left(\frac{x}{\varepsilon}, \partial_{x} u^{\varepsilon}\right)=0, \\
u^{\varepsilon}(x, 0)=f_{\varepsilon}(x) .
\end{gathered}
$$

Gonzalo Contreras was partially supported by CONACYT, Mexico, grant 178838, Antonio Siconolfi was partially supported by FP7-PEOPLE-2010-ITN, SADCO project, GA number 264735- SADCO. 
If $f_{\varepsilon}$ is continuous on $\mathbb{R}^{n}$ with linear growth, from [3], 4], 7] we know that there is a unique viscosity solution of the problem (1)-(2). Lions, Papanicolaou and Varadhan prove in [8] that if $f_{\varepsilon} \rightarrow f$ uniformly when $\varepsilon \rightarrow 0$ and $f$ is continuous with linear growth, the solutions $u^{\varepsilon}$ converge locally uniformly to the unique viscosity solution of the problem (3)-(4):

$$
\begin{gathered}
\partial_{t} u+\bar{H}\left(\partial_{x} u\right)=0, \\
u(x, 0)=f(x)
\end{gathered}
$$

where $\bar{H}$ is a convex hamiltonian which does not depend on $x$ and is called the effective Hamiltonian. We shall see below several characterizations of the effective Hamiltonian $\bar{H}$.

Equation (1) is seen as the Hamilton-Jacobi equation for a modified hamiltonian

$$
H_{\varepsilon}(x, p):=H\left(\frac{x}{\varepsilon}, p\right) .
$$

And it is said that $H_{\varepsilon} \rightarrow \bar{H}$ in the sense that the solutions of their Hamilton-Jacobi equations converge as stated by the homogenization result. The homogenization is interpreted as the convergence of solutions of Hamilton-Jacobi equations when the space is "seen from far away". Alternatively, one says that the limiting problems have a rapidly oscillating variable $\frac{x}{\varepsilon}$ which is "averaged" by the homogenization limit.

The effective Hamiltonian $\bar{H}$ is usually highly non-differentiable, but the solutions of the problem (3)-(4) are easily written because the characteristic curves for the equation (3) are the straight lines, and $p=\partial_{x} u$ is constant along them. Thus

$$
u(y, t)=\min _{x \in \mathbb{R}^{n}}\left\{f(x)+t \bar{L}\left(\frac{y-x}{t}\right)\right\},
$$

where

$$
\bar{L}(v):=\max _{p \in \mathbb{R}^{n}}[p(v)-\bar{H}(p)]
$$

is the effective Lagrangian. The simplicity of this limit solution and in general the possibility of using coarse grids in numerical analysis for the averaged problem are the main advantages of the homogenization in applications.

It turns out that the effective Lagrangian $\bar{L}$ is Mather's minimal action functional $\beta$ and the effective Hamiltonian $\bar{H}$ is Mather's alpha function, the convex dual of $\beta$. We recall the construction of the beta function below in (23). For now, it is interesting to observe that $\beta=\bar{L}$ is defined in the homology group $H_{1}\left(\mathbb{T}^{n}, \mathbb{R}\right)$, but in $(6)$ it is applied on velocity vectors. Similarly $\alpha=\bar{H}$ is defined in the cohomology group $H^{1}\left(\mathbb{T}^{n}, \mathbb{R}\right)$ and in equation (3) it is applied on gradients $\partial_{x} u$. This can be done because in the case of the torus $\mathbb{T}^{n}$ the groups $H_{1}\left(\mathbb{T}^{n}, \mathbb{R}\right) \approx \mathbb{R}^{n} \approx H^{1}\left(\mathbb{T}^{n}, \mathbb{R}\right)$ can be identified with the second factor in the trivial bundle $T \mathbb{T}^{n}=\mathbb{T}^{n} \times \mathbb{R}^{n}$. 
Mather's alpha function $\alpha=\bar{H}$ is related with Mañé's critical value [9] by ${ }^{1}$ $\alpha(P)=c(L-P)$. As such, the effective Hamiltonian $\bar{H}=\alpha$ has several interpretations, see [1], we name some of them:

(i) $\alpha$ is the convex dual of $\beta$.

(ii) $\alpha(P)=\inf \left\{k \in \mathbb{R} \mid \oint_{\gamma}(L-P+k) \geq 0 \quad \forall\right.$ closed curve $\gamma$ in $\left.\mathbb{T}^{n}\right\}$.

(iii) $\alpha(P)=\inf \left\{k \in \mathbb{R} \mid \Phi_{k}>-\infty\right\}$, where $\Phi_{k}: M \times M \rightarrow \mathbb{R}$ is

$$
\Phi_{k}(x, y)=\inf \left\{\oint_{\gamma}(L-P+k) \mid \gamma \text { curve in } \mathbb{T}^{n} \text { from } x \text { to } y\right\} .
$$

(iv) $\alpha(P)=-\inf \left\{\int(L-P) d \mu \mid \mu\right.$ is an invariant measure for $\left.L\right\}$.

(v) $\alpha(P)$ is the energy of the invariant measures $\mu$ which minimize $\int(L-P) d \mu$.

(vi) From Fathi's weak KAM theory [6], [5], $\alpha(P)$ is the unique constant for which there are global viscosity solutions of the Hamilton-Jacobi equation

$$
H\left(x, P+\partial_{x} v\right)=\alpha(P), \quad x \in \mathbb{T}^{n} .
$$

(vii) From [2], $\alpha(P)=\min _{u \in C^{1}\left(\mathbb{T}^{n}, \mathbb{R}\right)} \max _{x \in \mathbb{T}^{n}} H\left(x, P+\partial_{x} u\right)$.

(viii) From [2], $\alpha(P)$ is the minimum of energy levels which contain a lagrangian graph in $T^{*} \mathbb{T}^{n}$ with cohomology class $P$.

Following [8], knowing that the homogenization theorem holds, it is easy to prove that $\bar{H}=\alpha$. Indeed, consider the special case of affine initial conditions, $a \in \mathbb{R}^{n}, P \in\left(\mathbb{R}^{n}\right)^{*}$,

$$
f(x)=u(x, 0)=a+P \cdot x .
$$

The solution of

$$
u_{t}+\alpha\left(\partial_{x} u\right)=0
$$

with initial condition $(8)$ is

$$
u(x, t)=a+P \cdot x-\alpha(P) t .
$$

Using vi], fix a $\mathbb{Z}^{n}$-periodic viscosity solution of the Hamilton-Jacobi equation

$$
H\left(x, P+\partial_{x} v\right)=\alpha(P)
$$

given by the weak KAM theorem in [6]. Let

$$
u^{\varepsilon}(x, t)=u(x, t)+\varepsilon v\left(\frac{x}{\varepsilon}\right) .
$$

Define $f_{\varepsilon}$ by $u^{\varepsilon}(x, 0)=f_{\varepsilon}(x)$. Then $u^{\varepsilon}$ solves the problem (1)-(2). Since $f_{\varepsilon} \rightarrow f$ and $u^{\varepsilon} \rightarrow u$ uniformly, equation (9) must be the Hamilton-Jacobi equation for the effective Hamiltonian and hence $\bar{H}(P)=\alpha(P)$.

\footnotetext{
${ }^{1}$ Here $(L-P)(x, v):=L(x, v)-P \cdot v$
} 
Let $M$ be a compact path-connected riemannian manifold without boundary. A Tonelli hamiltonian on $M$ is a $C^{2}$ function $H: T^{*} M \rightarrow \mathbb{R}$ on the cotangent bundle $T^{*} M$ which is convex and superlinear as in (b), (c) above. We want to generalize the Lions-PapanicolaouVaradhan Theorem to this setting. The generalization of their theorem to other compact manifolds has three problems, namely

(1) It is not clear how to choose the generalization of $\frac{x}{\varepsilon}$.

(2) In the modified hamiltonian $H_{\varepsilon}$ in (5) the base point changes to $\frac{x}{\varepsilon}$ but the moment $p$ "remains the same". It is not clear how to do this in non-parallelizable manifolds. Similarly, the effective Hamiltonian $\bar{H}(P)$ "does not depend on $x$ ". Again, this is not natural if the manifold is not parallelizable.

(3) Mather's alpha function, the candidate for the effective Hamiltonian, is defined on the first cohomology group $\alpha: H^{1}(M, \mathbb{R}) \approx \mathbb{R}^{k} \rightarrow \mathbb{R}$, which may not be a cover of the manifold. Thus the (limiting) effective Hamiltonian and the Hamilton-Jacobi equations for $H_{\varepsilon}$ would be defined on very different spaces. In particular, these spaces usually have different dimensions.

To solve the last problem we will use an ad hoc definition of convergence of spaces very much inspired by the Gromov Hausdorff convergence. For the second problem, a change of variables in the torus allows to change the parameter $\varepsilon$ in the space variables $\frac{x}{\varepsilon}$ to the momentum variables. Indeed, write

$$
u^{\varepsilon}(x, t)=v^{\varepsilon}\left(\frac{x}{\varepsilon}, t\right)
$$

Then the problem (1)-(2) for $v^{\varepsilon}(y, t)$ becomes

$$
\begin{gathered}
\partial_{t} v^{\varepsilon}+H\left(y, \frac{1}{\varepsilon} \partial_{y} v^{\varepsilon}\right)=0 \\
v^{\varepsilon}(y, 0)=f_{\varepsilon}(\varepsilon y) .
\end{gathered}
$$

Observe that now equation (14) makes sense in any manifold, but equation (15) does not. We will take care of this later.

Given a metric space $(\mathcal{M}, \mathrm{d})$, a family of metric spaces $\left(\mathcal{M}_{n}, \mathrm{~d}_{n}\right)$ and continuous maps $F_{n}:\left(\mathcal{M}_{n}, \mathrm{~d}_{n}\right) \rightarrow(\mathcal{M}, \mathrm{d})$, we give a notion of convergence of $\mathcal{M}_{n}$ to $\mathcal{M}$ through $F_{n}, F_{n}$ should be interpreted as a telescope through which we look at the limit space $\mathcal{M}$ from $\mathcal{M}_{n}$. The definition basically requires equivalence of distances $\mathrm{d}_{n}$ and the pullbacks of $\mathrm{d}$ under $F_{n}$, with adjustments to cope with the case where $F_{n}$ is not injective and/or not surjective. We will say that $\lim _{n}\left(\mathcal{M}_{n}, \mathrm{~d}_{n}, F_{n}\right)=(\mathcal{M}$, d) if

(a) There are $K>1$ and $A_{n}>0$ such that $\lim _{n} A_{n}=0$ with

$$
\forall x, y \in \mathcal{M}_{n}, \quad K^{-1} \mathrm{~d}_{n}(x, y)-A_{n} \leq \mathrm{d}\left(F_{n}(x), F_{n}(y)\right) \leq K \mathrm{~d}_{n}(x, y) .
$$

(b) For any $y \in \mathcal{M}$ there is a sequence $x_{n} \in \mathcal{M}_{n}$ such that $\lim _{n} F_{n}\left(x_{n}\right)=y$. 
Observe that condition (b) implies that for any metric ball $\mathbb{K}$ of $\mathcal{M}$

$$
\mathbb{K} \cap F_{n}\left(\mathcal{M}_{n}\right) \neq \emptyset \text { for } n \text { sufficiently large, }
$$

a kind of surjectivity statement. Similarly, using the convention diam $\emptyset=0$, condition (a) implies that $\lim _{n} \operatorname{diam} F_{n}^{-1}\{y\}=0$ for all $y \in M$.

If $\lim _{n}\left(\mathcal{M}_{n}, \mathrm{~d}_{n}, F_{n}\right)=(\mathcal{M}, \mathrm{d})$ and $f_{n}: \mathcal{M}_{n} \rightarrow \mathbb{R}, f: \mathcal{M} \rightarrow \mathbb{R}$, we say that $f_{n}$ locally uniformly $F_{n}$-converges to $f$ if for every $x \in \mathcal{M}$ and subsequences $y_{n_{k}} \in \mathcal{M}_{n_{k}}$ with $\lim _{n_{k}} F_{n_{k}}\left(y_{n_{k}}\right)=x$, one has $\lim _{n_{k}} f_{n_{k}}\left(y_{n_{k}}\right)=f(x)$.

If $\lim _{n}\left(\mathcal{M}_{n}, \mathrm{~d}_{n}, F_{n}\right)=(\mathcal{M}, \mathrm{d})$ and $f_{n}: \mathcal{M}_{n} \rightarrow \mathbb{R}, f: \mathcal{M} \rightarrow \mathbb{R}$, we say that $f_{n}$ uniformly $F_{n}$-converges to $f$ if it locally uniformly $F_{n}$-converges to $f$ and in addition

$$
\lim _{n} \sup _{x \in \mathcal{M}_{n}}\left|f_{n}(x)-f\left(F_{n}(x)\right)\right|=0 .
$$

The initial Hamiltonian will be the lift of $H$ to the maximal free abelian cover of $M$ defined as follows. Let $\widetilde{M}$ be the covering space of $M$ defined by $\pi_{1}(\widetilde{M})=$ ker $\mathfrak{h}$, where $\mathfrak{h}: \pi_{1}(M) \rightarrow H_{1}(M, \mathbb{Z})$ is the Hurewicz homomorphism. Its group of deck transformations is $\mathbb{G}=\operatorname{im}\left[\pi_{1}(M) \rightarrow H_{1}(M, \mathbb{Z})\right]$, which is a free abelian group, $\mathbb{G} \approx \mathbb{Z}^{k} \subset H_{1}(M, \mathbb{R}) \approx \mathbb{R}^{k}$. Observe that the large-scale structure of the covering space $\widetilde{M}$ is given by $\mathbb{G}=\mathbb{Z}^{k}$. In the homogenized problem the position space, or configuration space, is the homology group $x \in H_{1}(M, \mathbb{R}) \approx \mathbb{R}^{k}$, and the momenta, and the derivatives $\partial_{x} u$, are in its dual, the cohomology group $\left\{p, \partial_{x} u\right\} \subset H^{1}(M, \mathbb{R}) \approx \mathbb{R}^{k}$.

Let $d$ be the metric on $\widetilde{M}$ induced by the lift of the riemannian metric on $M$, set for any $\varepsilon>0, d_{\varepsilon}:=\varepsilon d$. Then $\left(\widetilde{M}, d_{\varepsilon}\right)$ converges to $H_{1}(M, \mathbb{R})$ in the same way as $\varepsilon \mathbb{Z}^{k}$ converges to $\mathbb{R}^{k}$ or $\varepsilon \mathbb{G} \stackrel{\varepsilon}{\longrightarrow} H_{1}(M, \mathbb{R})$.

To be precise, fix a basis $c_{1}, \ldots, c_{k}$ of $H^{1}(M, \mathbb{R})$ and fix closed 1 -forms $\omega_{1}, \ldots, \omega_{k}$ in $M$ such that $\omega_{i}$ has cohomology class $c_{i}$. By the universal coefficient theorem $H_{1}(M, \mathbb{R})=$ $H^{1}(M, \mathbb{R})^{*}$. Let $G: \widetilde{M} \rightarrow H_{1}(M, \mathbb{R})$ be given by

$$
G(x) \cdot\left(\sum_{i} a_{i} c_{i}\right)=\oint_{x_{0}}^{x}\left(\sum_{i} a_{i} \widetilde{\omega}_{i}\right),
$$

where $x_{0}$ is a base point in $\widetilde{M}$ and $\widetilde{\omega}_{i}$ is the lift of $\omega_{i}$ to $\widetilde{M}$. Since $\widetilde{\omega}$ is exact, the integral does not depend on the choice of the path from $x_{0}$ to $x$. Notice that the function $G$ depends on the choice of $x_{0}$, on the choice of the basis $\left\{c_{i}\right\}$ and of representatives $\omega_{i}$ in $c_{i}$. However, we shall be interested in the functions $\varepsilon G$ for $\varepsilon$ small, and these dependencies disappear in the limit $\varepsilon \rightarrow 0$. On the points in $\widetilde{M}$ in the same fiber as $x_{0}$ the value of $G$ does not depend on the chosen basis $\left\{\omega_{i}\right\}$ because in that case it is an integral on a closed curve in $M$. In general, if $x, y$ belong to the same fiber, then $G(x)-G(y)$ is the transformation in $\mathbb{G}=\operatorname{im}\left[\pi_{1}(M) \rightarrow H_{1}(M, \mathbb{Z})\right]$ carrying $y$ to $x$, condition which uniquely 
identifies it, conversely, any deck transformation admits a representation of this type. We set $F_{\varepsilon}=\varepsilon G$.

1.1. Proposition. For the maximal free abelian cover we have that

$$
\lim _{\varepsilon}\left(\widetilde{M}, d_{\varepsilon}, F_{\varepsilon}\right)=H_{1}(M, \mathbb{R})
$$

We finally address the problem of initial conditions. If we want to mimic the torus case where the same initial datum can be taken for approximating as well as for limit equations, we can think of transferring a continuous datum $f$ defined in $H_{1}(M, \mathbb{R})$ to $\widetilde{M}$ by setting $f_{\varepsilon}(y)=f\left(F_{\varepsilon}(y)\right)$, which is interpreted as $f$ "seen" on $\left(\widetilde{M}, d_{\varepsilon}\right)$. We recognize in the relationship between $f$ and $f_{\varepsilon}$ a special instance of uniform $F_{\varepsilon}$-convergence, and this is indeed the way in which the matter will be presented in the forthcoming statement of the main homogenization result. The proof of this theorem will be given in Section 3 .

\subsection{Theorem.}

Let $H: T^{*} M \rightarrow \mathbb{R}$ be a Tonelli hamiltonian on a compact manifold $M$. Let $f_{\varepsilon}: \widetilde{M} \rightarrow \mathbb{R}, f: H_{1}(M, \mathbb{R}) \rightarrow \mathbb{R}$ be continuous with $f$ of at most linear growth, assume that $f_{\varepsilon}$ uniformly $F_{\varepsilon}$-converges to $f$. Let $\widetilde{H}: T^{*} \widetilde{M} \rightarrow \mathbb{R}$ be the lift of $H$ to $\widetilde{M}$. Let $v^{\varepsilon}$ be the viscosity solution to the problem

$$
\begin{gathered}
\partial_{t} v^{\varepsilon}+\widetilde{H}\left(x, \frac{1}{\varepsilon} \partial_{x} v^{\varepsilon}\right)=0, \quad x \in \widetilde{M}, t>0 \\
v^{\varepsilon}(x, 0)=f_{\varepsilon}(x) .
\end{gathered}
$$

Then the family of functions $v^{\varepsilon}: \widetilde{M}_{\varepsilon} \times\left[0,+\infty\left[\rightarrow \mathbb{R}\right.\right.$ locally uniformly $F_{\varepsilon}$-converges in $\widetilde{M} \times] 0,+\infty\left[{ }^{2}\right.$ to the viscosity solution $u: H_{1}(M, \mathbb{R}) \times[0,+\infty[\rightarrow \mathbb{R}$ of the problem

$$
\begin{aligned}
& \partial_{t} u+\bar{H}\left(\partial_{h} u\right)=0, \quad h \in H_{1}(M, \mathbb{R}), t>0 ; \\
& u(h, 0)=f(h)
\end{aligned}
$$

where the effective Hamiltonian $\bar{H}$ is Mather's alpha function $\bar{H}=\alpha: H^{1}(M, \mathbb{R}) \rightarrow \mathbb{R}$.

Several comments are still in order:

1) The convergence destroys the differential structure of the spaces. Nevertheless we obtain convergence of solutions $v^{\varepsilon}$ to a solution of a partial differential equation on the limit space because the Hamilton-Jacobi equation is an encoding of a variational principle. Namely, its solutions are the minimal cost functions under the Lagrangian. This variational principle is preserved under the limit of spaces.

2) In this setting it is possible to prove the homogenization theorem using standard methods. This is very good news since we expect that many homogenization results generalize from the torus to other manifolds. However, using a result of Mather, we will provide another proof, which is essentially a change of variables in the Lax formula.

\footnotetext{
${ }^{2}$ Our proof of the uniformity does not extend to $t=0$.
} 
3) Motivated by possible applications we extend the result to other Abelian covers.

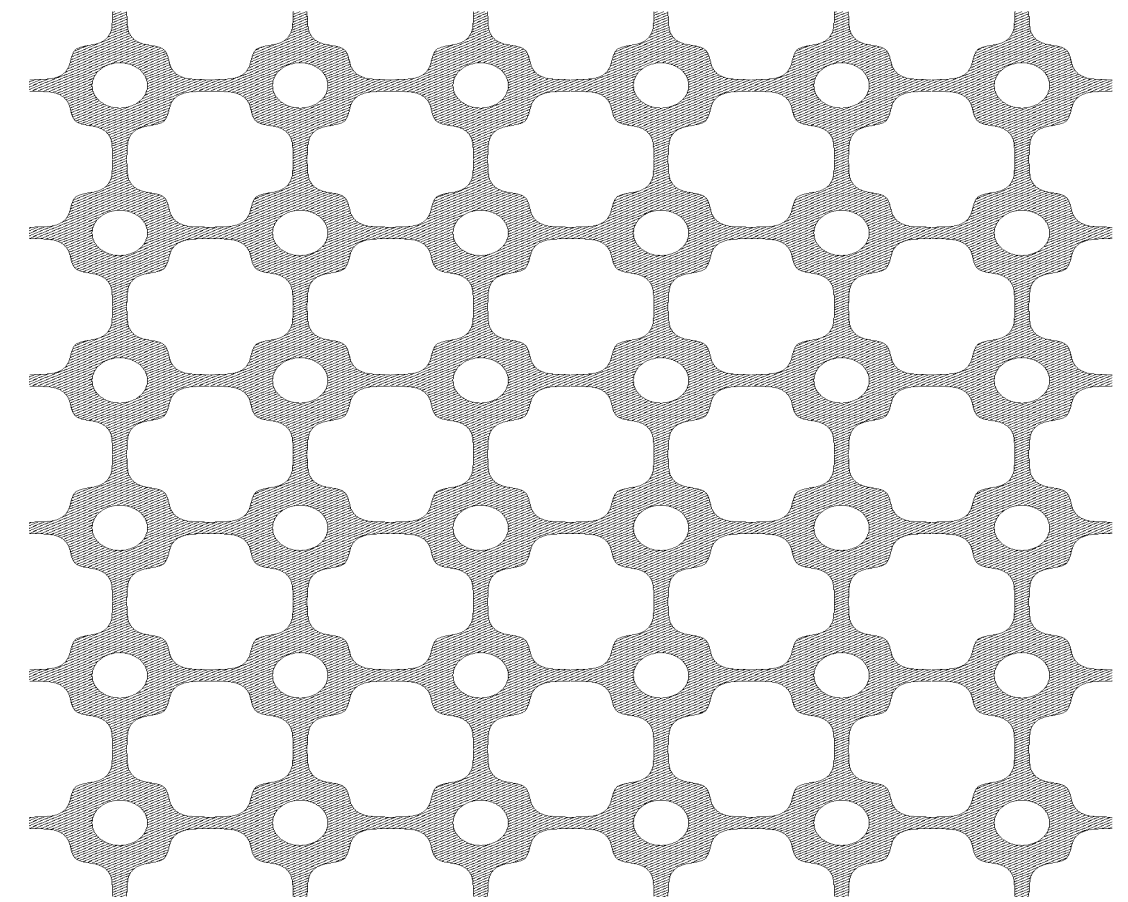

Figure 1. A free abelian (sub)cover of the compact orientable surface of genus 3 with group of covering transformations $\mathbb{Z}^{2}$.

\subsection{Subcovers.}

For general abelian covers, i.e. covering spaces whose group of deck transformations $\mathbb{D}$ is abelian, the torsion part of $\mathbb{D}$ is killed under the limit of $\widehat{M}_{\varepsilon}=(\widehat{M}, \varepsilon \widehat{d})$. Thus the limit is the same as for a free abelian cover, where $\mathbb{D}$ is free abelian. These coverings are subcovers of the maximal free abelian cover $\widetilde{M}$. In this case we have similar results as in Theorem 1.2

Let $L: T M \rightarrow \mathbb{R}$ be the lagrangian of $H$ i.e.

$$
L(x, v):=\max _{p \in T_{x}^{*} M}[p(v)-H(x, p)] .
$$

The Euler-Lagrange equation for $L$ is

$$
\frac{d}{d t} \partial_{v} L=\partial_{x} L
$$

It determines a complete flow $\varphi$ on $T M$ by $\varphi_{t}(x, v)=(\gamma(t), \dot{\gamma}(t))$ where $\gamma$ is the solution of 22 with $(\gamma(0), \dot{\gamma}(0))=(x, v)$. Given an invariant Borel probability $\mu$ for $\varphi_{t}$ with compact support define its homology class $\rho(\mu) \in H_{1}(M, \mathbb{R})=H^{1}(M, \mathbb{R})^{*}$ by

$$
\rho(\mu) \cdot c=\int_{T M} \omega d \mu
$$


where $\omega$ is any closed 1-form on $M$ with cohomology class $c$. Mather's minimal action function is $\beta: H_{1}(M, \mathbb{R}) \rightarrow \mathbb{R}$,

$$
\beta(h):=\inf _{\rho(\mu)=h} \int L d \mu
$$

where the infimum is over all $\varphi_{t}$-invariant probabilities with homology $\rho(\mu)=h$.

Free abelian covers $\widehat{M}$ are obtained from normal subgroups of the fundamental group $\pi_{1}(M)$ containing the commutators. If $\mathfrak{g}$ is the canonical epimorphism from $\pi_{1}(M)$ to the quotient group then $\pi_{1}(\widehat{M})=$ ker $\mathfrak{g}$ and the group of deck transformations is im $\mathfrak{g}$. It can be identified to $\mathbb{Z}^{\ell}$, up to the choice of a basis. Since $H_{1}(M, \mathbb{Z})$ is the abelianization of $\pi_{1}(M)$, such epimorphism $\mathfrak{g}$ factors as $\mathfrak{g}=\mathfrak{f} \circ \mathfrak{h}$ with $\mathfrak{g}: \pi_{1}(M) \stackrel{\mathfrak{h}}{\longrightarrow} H_{1}(M, \mathbb{Z}) \stackrel{\mathfrak{f}}{\longrightarrow}$ im $\mathfrak{g}$. The linearization of $\mathfrak{f}$ gives a linear epimorphism $\mathfrak{f}: H_{1}(M, \mathbb{R}) \rightarrow \operatorname{im} \mathfrak{f} \approx \mathbb{R}^{\ell}$. Loosely speaking, the elements of im $\mathfrak{f}$ can be interpreted as homology classes adapted to the cover $\widehat{M}$. We denote by $\pi$ the covering projection of $\widetilde{M}$ onto $\widehat{M}$. We record for later use that, given any norm on $H_{1}(M, \mathbb{R}), H_{1}(M, \mathbb{Z}) \cap \operatorname{ker} \mathfrak{f} \approx \mathbb{Z}^{k-\ell}$ is $B$-dense in ker $\mathfrak{f} \approx \mathbb{R}^{k-\ell}$ for a suitable constant $B$. Further, for any fixed $x_{0} \in \widetilde{M}$

$$
H_{1}(M, \mathbb{Z}) \cap \operatorname{ker} \mathfrak{f}=\left\{G(x)-G\left(x_{0}\right) \mid \pi(x)=\pi\left(x_{0}\right)\right\} .
$$

The metric on $\widehat{M}$, denoted by $\widehat{d}$, induced by the lift of the riemannian metric on $M$, is given by

$$
\widehat{d}(x, y)=\inf \left\{d(z, w) \mid z \in \pi^{-1}(x), w \in \pi^{-1}(y)\right\}
$$

The minimal action functional for the cover $\widehat{M}$ is $\widehat{\beta}: \mathbb{R}^{\ell}=\operatorname{im} \mathfrak{f} \rightarrow \mathbb{R}$,

$$
\widehat{\beta}(z)=\inf \{\beta(h) \mid \mathfrak{f}(h)=z\} .
$$

This can also be interpreted as the average action of minimizing Euler-Lagrange orbits on $\widehat{M}$ with asymptotic direction $z$. The effective Hamiltonian for $\widehat{M}$ is $\bar{H}=\widehat{\beta}^{*}$, the convex dual of $\widehat{\beta}$ :

$$
\begin{aligned}
\bar{H}(p)=\widehat{\beta}^{*}(p) & =\max _{z \in \operatorname{im} \mathfrak{f}} p(z)-\widehat{\beta}(z) \\
& =\max _{h \in H_{1}(M, \mathbb{R})} p(\mathfrak{f}(h))-\beta(h) \\
& =\alpha\left(\mathfrak{f}^{*}(p)\right),
\end{aligned}
$$

where $\mathfrak{f}^{*}$ is the homomorphism $\mathfrak{f}^{*}:(\operatorname{im} \mathfrak{f})^{*} \rightarrow H_{1}(M, \mathbb{R})^{*}=H^{1}(M, \mathbb{R})$ induced by $\mathfrak{f}$.

By (24)

$$
\mathfrak{f}(G(y))=\mathfrak{f}(G(x)) \quad \text { whenever } \pi(x)=\pi(y) .
$$


Then $\mathfrak{f}(G(\cdot))$ is the lift to $\widetilde{M}$ of a map from $\widehat{M}$ to $\operatorname{im} \mathfrak{f}$ that we denote by $\widehat{G}$. We set, for any $\varepsilon>0, \widehat{F}_{\varepsilon}=\varepsilon \widehat{G}$.

\subsection{Proposition.}

$$
\lim _{\varepsilon}\left(\widehat{M}, \varepsilon \widehat{d}, \varepsilon \widehat{F}_{\varepsilon}\right)=\operatorname{im} \mathfrak{f}
$$

The proof will be given in the next section. We proceed to give the statement of the homogenization result for general abelian covers, the proof will be provided in Section 4 .

\subsection{Theorem.}

Let $f_{\varepsilon}: \widehat{M} \rightarrow \mathbb{R}, f: \operatorname{im} \mathfrak{f} \rightarrow \mathbb{R}$ be continuous with $f$ of linear growth. Assume that $f_{\varepsilon}$ uniformly $\widehat{F}_{\varepsilon}$-converges to $f$. Let $\widehat{H}: T^{*} \widehat{M} \rightarrow \mathbb{R}$ be the lift of a Tonelli Hamiltonian $H$, defined on $M$, to $\widehat{M}$. Let $v^{\varepsilon}$ be the viscosity solution to the problem

$$
\begin{gathered}
\partial_{t} v^{\varepsilon}+\widehat{H}\left(x, \frac{1}{\varepsilon} \partial_{x} v^{\varepsilon}\right)=0, \quad x \in \widehat{M}, t>0 ; \\
v^{\varepsilon}(x, 0)=f_{\varepsilon}(x) .
\end{gathered}
$$

Then the family of functions $v^{\varepsilon}: \widehat{M}_{\varepsilon} \times\left[0,+\infty\left[\rightarrow \mathbb{R}\right.\right.$ locally uniformly $\widehat{F}_{\varepsilon}$-converges in $\widehat{M} \times] 0,+\infty[$ to the solution $u: \operatorname{im} \mathfrak{f} \times[0,+\infty[\rightarrow \mathbb{R}$ of the problem

$$
\begin{gathered}
\partial_{t} u+\bar{H}\left(\partial_{q} u\right)=0, \quad q \in \operatorname{im} \mathfrak{f}, t>0 ; \\
u(q, 0)=f(q) ;
\end{gathered}
$$

where the effective Hamiltonian $\bar{H}:(\mathrm{im} \mathfrak{f})^{*} \rightarrow \mathbb{R}$, is $\bar{H}=\mathfrak{f}^{*} \alpha$ given by (26).

\section{Convergence of Spaces}

In this section we provide proofs for Propositions 1.1, 1.3, and show that some basic properties holding for usual uniform convergences are still true in our setting.

\section{Proof of proposition 1.1;}

Observe that for the finite dimensional space $H_{1}(M, \mathbb{R})$ we can use any norm $\|\cdot\|$.

If $\omega=\sum_{i} a_{i} \omega_{i}$ and $\|\omega\|:=\sup _{x \in M}|\omega(x)|$,

$$
|[G(x)-G(y)] \cdot[\omega]|=\left|\oint_{x}^{y} \widetilde{\omega}\right| \leq\|\omega\| d(x, y) .
$$

Then there is $K_{0}>0$ such that

$$
|G(x)-G(y)| \leq K_{0} d(x, y),
$$

and using that $F_{\varepsilon}=\varepsilon G$ and $d_{\varepsilon}=\varepsilon d$, we have that

$$
\left\|F_{\varepsilon}(x)-F_{\varepsilon}(y)\right\| \leq K_{0} d_{\varepsilon}(x, y) .
$$


Write $\mathbb{G}=\operatorname{im}\left[\pi_{1}(M) \rightarrow H_{1}(M, \mathbb{R})\right]$, the group of covering transformations of $\widetilde{M}$. Fix $x_{0} \in \widetilde{M}$. Let $e_{1}, \ldots, e_{k}$ be a basis of $\mathbb{G}$ and let $\widehat{\gamma}_{i}$ be a minimal geodesic from $x_{0}$ to $e_{i}\left(x_{0}\right)=: x_{0}+e_{i}$. If $\pi: \widetilde{M} \rightarrow M$ is the projection, the concatenation $\left(\pi \circ \widehat{\gamma}_{1}\right)^{n_{1}} * \cdots *\left(\pi \circ \widehat{\gamma}_{k}\right)^{n_{k}}$ lifts to a curve from $x_{0}$ to $x_{0}+\bar{n}$, where $\bar{n}=\sum n_{i} e_{i}$. Let $\ell_{i}$ be the length of $\gamma_{i}$. Then

$$
d\left(x_{0}, x_{0}+\bar{n}\right) \leq \sum_{i} n_{i} \ell_{i} \leq\left(\max _{i} \ell_{i}\right) k\|\bar{n}\|=: K_{2}\|\bar{n}\|
$$

For any $\bar{n}, \bar{m} \in \mathbb{G} \approx \mathbb{Z}^{k}$, we have

$$
d\left(x_{0}+\bar{n}, x_{0}+\bar{m}\right)=d\left(x_{0}, x_{0}+(\bar{n}-\bar{m})\right) \leq K_{2}\|\bar{n}-\bar{m}\| .
$$

If $x, y \in \widetilde{M}$ there are two elements $x_{0}+\bar{n}, x_{0}+\bar{m}$ of the orbit of $x_{0}$ such that $d\left(x, x_{0}+\bar{n}\right) \leq D$ and $d\left(y, x_{0}+\bar{m}\right) \leq D$, where $D:=\operatorname{diam} M$. We have that

$$
\begin{aligned}
d(x, y) & \leq d\left(x, x_{0}+\bar{n}\right)+d\left(x_{0}+\bar{n}, x_{0}+\bar{m}\right)+d\left(x_{0}+\bar{m}, y\right) \\
& \leq K_{2}\|\bar{n}-\bar{m}\|+2 D .
\end{aligned}
$$

Observe that

$$
G\left(x_{0}+\bar{m}\right)-G\left(x_{0}+\bar{n}\right)=\bar{m}-\bar{n} \in H_{1}(M, \mathbb{R}) .
$$

Using the Lipschitz property 32 for $G$,

$$
\begin{aligned}
|G(x)-G(y)| & \geq\|\bar{m}-\bar{n}\|-\left\|G(x)-G\left(x_{0}+\bar{n}\right)\right\|-\left\|G(y)-G\left(x_{0}+\bar{m}\right)\right\| \\
& \geq\|\bar{m}-\bar{n}\|-2 K_{0} D .
\end{aligned}
$$

Therefore

$$
d(x, y) \leq K_{2}\|G(x)-G(y)\|+2 K_{0} K_{2} D+2 D .
$$

For $A:=2 K_{0} D+2 K_{2}^{-1} D$,

$$
\forall x, y \in \widetilde{M}, \quad K_{2}^{-1} d(x, y)-A \leq\|G(x)-G(y)\| .
$$

Multiplying the inequality by $\varepsilon$, we get

$$
\forall x, y \in \widetilde{M}, \quad K_{2}^{-1} d_{\varepsilon}(x, y)-\varepsilon A \leq\left\|F_{\varepsilon}(x)-F_{\varepsilon}(y)\right\| .
$$

Inequalities (33) and (35) prove condition (a) of the convergence.

Condition (b) follows from the fact that the image of the $\mathbb{G}$-orbit of $x_{0}$,

$$
F_{\varepsilon}\left(x_{0}+\mathbb{G}\right)=\varepsilon \mathbb{G}=\varepsilon \mathbb{Z}^{k} \subset \mathbb{R}^{k}=H_{1}(M, \mathbb{R}),
$$

is $\varepsilon$-dense in $H_{1}(M, \mathbb{R})$.

\section{Proof of proposition 1.3}

We endow im $\mathfrak{f}$ with the norm $\|\cdot\|_{\mathfrak{f}}$ defined as

$$
\|q\|_{\mathfrak{f}}=\min \left\{\|h\| \mid h \in H_{1}(M, \mathbb{R}) \text { with } \mathfrak{f}(h)=q\right\},
$$

where $\|\cdot\|$ is any norm for $H_{1}(M, \mathbb{R})$. 
Let $x, y$ be in $\widehat{M}$ and $z, w$ any element in $\pi^{-1}(x), \pi^{-1}(y)$, respectively. Exploiting the convergence proved in Proposition 1.1, we have

$$
\|\widehat{G}(x)-\widehat{G}(y)\|_{\mathfrak{f}}=\|\mathfrak{f}(G(z)-G(w))\|_{\mathfrak{f}} \leq\|G(z)-G(w)\| \leq k d(z, w),
$$

which implies, by arbitrariness of $z, w$

$$
\|\widehat{G}(x)-\widehat{G}(y)\|_{\mathfrak{f}} \leq k \widehat{d}(x, y) .
$$

By $B$-density of $H_{1}(M, \mathbb{Z}) \cap \operatorname{ker} \mathfrak{f}$ in $\operatorname{ker} \mathfrak{f}$ and $(24)$, we find $z \in \pi^{-1}(x), w \in \pi^{-1}(y)$ with

$$
\|\widehat{G}(x)-\widehat{G}(y)\|_{\mathfrak{f}} \geq\|G(z)-G(w)\|-B
$$

from this we derive, again using Proposition 1.1

$$
\|\widehat{G}(x)-\widehat{G}(y)\|_{\mathfrak{f}} \geq k^{-1} d(z, w)-A-B \geq k^{-1} \widehat{d}(y, x)-A-B .
$$

The last inequality together with (36) gives, up to multiplying by $\varepsilon$, the first property of the convergence. Given $q=\mathfrak{f}(h) \in \operatorname{im} \mathfrak{f}$, we know that there is a sequence $x_{\varepsilon}$ in $\widetilde{M}$ with $\varepsilon G\left(x_{\varepsilon}\right)$ converging to $h$, therefore

$$
q=\lim _{\varepsilon} \varepsilon \mathfrak{f}\left(G\left(x_{\varepsilon}\right)\right)=\lim _{\varepsilon} \varepsilon \widehat{G}\left(\pi\left(x_{\varepsilon}\right)\right) .
$$

This concludes the proof.

We go back to the abstract setting considering general metric spaces $\mathcal{M}, \mathcal{M}_{n}$, we will assume from now on the limit space $\mathcal{M}$ to be locally compact, namely we require

All the metric balls of $\mathcal{M}$ are relatively compact.

This will fit the frame of homogenization. To avoid pathological cases, we also make clear that the compact subsets of $\mathcal{M}$ we will consider in what follows, usually denoted by $\mathbb{K}$, are understood, without further mentioning, to be with nonempty interior. This will guarantee that $F_{n}^{-1}(\mathbb{K}) \neq \emptyset$, at least for $n$ large, thanks to 16 .

If $\lim _{n}\left(\mathcal{M}_{n}, \mathrm{~d}_{n}, F_{n}\right)=(\mathcal{M}, \mathrm{d})$ and $f_{n}: \mathcal{M}_{n} \rightarrow \mathbb{R}$, we say that the family $f_{n}$ is $F_{n^{-}}$ locally equicontinuous if for any compact subset $\mathbb{K}$ of $\mathcal{M}$ and any $\varepsilon>0$, there exists $\delta=\delta(\varepsilon, \mathbb{K})>0$ such that

$$
x, y \in F_{n}^{-1}(\mathbb{K}), \quad \mathrm{d}_{n}(x, y)<\delta \quad \Longrightarrow \quad\left|f_{n}(x)-f_{n}(y)\right|<\varepsilon .
$$

In accord to what the term uniform suggests, continuity is stable under the locally uniform $F_{n}$-convergence.

2.1. Proposition. Assume $\lim _{n}\left(\mathcal{M}_{n}, \mathrm{~d}_{n}, F_{n}\right)=(\mathcal{M}, \mathrm{d})$, and take $f_{n}: \mathcal{M}_{n} \rightarrow \mathbb{R}, f: \mathcal{M} \rightarrow$ $\mathbb{R}$ with $f_{n}$ locally uniformly $F_{n}$-convergent to $f$. If all the $f_{n}$ are continuous then $f$ is continuous. 
Proof: We claim that the $f_{n}$ are $F_{n}$-locally equicontinuous. In fact, if this were not true there should be, taking into account that all the $f_{n}$ are continuous, for a given compact $\mathbb{K}$ in $\mathcal{M}$ and $\delta>0,\left(\right.$ sub)sequences $z_{n} \in F_{n}^{-1}(\mathbb{K}), y_{n} \in F_{n}^{-1}(\mathbb{K})$ with

$$
\mathrm{d}_{n}\left(z_{n}, y_{n}\right) \rightarrow 0 \text { and }\left|f_{n}\left(y_{n}\right)-f_{n}\left(z_{n}\right)\right|>\delta .
$$

By condition (a) in the definition of spaces convergence $\mathrm{d}\left(F_{n}\left(x_{n}\right), F_{n}\left(z_{n}\right)\right) \rightarrow 0$, and so, owing to compactness of $\mathbb{K}, F_{n}\left(x_{n}\right)$ and $F_{n}\left(z_{n}\right)$ converge, up to subsequences, to the same element, say $x$, of $\mathcal{M}$. By the very definition of local uniform convergence, we deduce $\lim _{n} f_{n}\left(x_{n}\right)=\lim _{n} f_{n}\left(z_{n}\right)=f(x)$, which is in contrast with (37).

We proceed proving that $f$ is uniformly continuous in $\mathbb{K}$. Given $\varepsilon>0$ let $\delta=\delta(\varepsilon, \mathbb{K})>0$ be such that

$$
\forall n, \quad \forall y, z \in F_{n}^{-1}(\mathbb{K}), \quad \mathrm{d}_{n}(y, z)<\delta \Longrightarrow\left|f_{n}(y)-f_{n}(z)\right|<\varepsilon .
$$

Let $x_{0}, x_{1} \in \mathcal{M}$ with $\mathrm{d}\left(x_{0}, x_{1}\right)<\frac{\delta}{K}$, where $K$ is the constant appearing in condition (a) of the definition of spaces convergence. Let $z_{n}, y_{n} \in \mathcal{M}_{n}$ with $\lim _{n} F_{n}\left(z_{n}\right)=x_{0}$, $\lim _{n} F_{n}\left(y_{n}\right)=x_{1}$, then $\lim _{n} f_{n}\left(z_{n}\right)=f\left(x_{0}\right), \lim _{n} f_{n}\left(y_{n}\right)=f\left(x_{1}\right)$ and

$$
\mathrm{d}_{n}\left(z_{n}, y_{n}\right) \leq K \mathrm{~d}\left(F_{n}\left(z_{n}\right), F_{n}\left(y_{n}\right)\right)+A_{n} K \stackrel{n}{\longrightarrow} K \mathrm{~d}\left(x_{0}, x_{1}\right)<\delta .
$$

Thus

$$
\left|f_{n}\left(z_{n}\right)-f_{n}\left(y_{n}\right)\right|<\varepsilon
$$

Taking $\lim \sup _{n}$ on the inequality

$$
\left|f\left(x_{0}\right)-f\left(x_{1}\right)\right| \leq\left|f\left(x_{0}\right)-f_{n}\left(z_{n}\right)\right|+\left|f_{n}\left(z_{n}\right)-f_{n}\left(y_{n}\right)\right|+\left|f_{n}\left(y_{n}\right)-f\left(x_{1}\right)\right|,
$$

we obtain that

$$
\left|f\left(x_{0}\right)-f\left(x_{1}\right)\right| \leq \varepsilon
$$

as desired.

If $\lim _{n}\left(\mathcal{M}_{n}, d_{n}, F_{n}\right)=(\mathcal{M}, d)$, we say that a family of functions $f_{n}:\left(\mathcal{M}_{n}, d_{n}\right) \rightarrow \mathbb{R}$ converges pointwise to $f:(\mathcal{M}, d) \rightarrow \mathbb{R}$ if for every $x \in \mathcal{M}$ there are sequences $x_{n} \in \mathcal{M}_{n}$ with $\lim _{n} F_{n}\left(x_{n}\right)=x$ and $\lim _{n} f_{n}\left(x_{n}\right)=f(x)$.

We proceed deriving an result linking equicontinuity and local uniform convergence.

2.2. Proposition. Assume $\lim _{n}\left(\mathcal{M}_{n}, d_{n}, F_{n}\right)=(\mathcal{M}, d)$, take $f:(\mathcal{M}, d) \rightarrow \mathbb{R}$ and continuous functions $f_{n}:\left(\mathcal{M}_{n}, d_{n}\right) \rightarrow \mathbb{R}$. The family $f_{n}$ locally uniformly $F_{n}$-converges to $f$ if and only if it is pointwise convergent and $F_{n}$-locally equicontinuous.

Proof: The implication (local uniform convergence) $\Rightarrow$ (equicontinuity) has already been proved in Proposition 2.1, pointwise convergence can also be trivially derived. This shows one half of the statement. Conversely, assume that a subsequence, still indexed by $n$, $y_{n} \in \mathcal{M}_{n}$ satisfies $F_{n}\left(y_{n}\right)=x_{0}$, for some $x_{0} \in \mathcal{M}$, by pointwise convergence there is 
$z_{n} \in \mathcal{M}_{n}$ with $\lim _{n} F_{n}\left(z_{n}\right)=x_{0}, \lim _{n} f_{n}\left(z_{n}\right)=f\left(x_{0}\right)$, therefore $x_{n}, z_{n}$ belong to $F_{n}^{-1}(\mathbb{K})$, for a suitable compact subset $\mathbb{K} \subset \mathcal{M}$ and any $n$, in addition

$$
\mathrm{d}_{n}\left(y_{n}, z_{n}\right) \leq K \mathrm{~d}\left(F_{n}\left(y_{n}\right), F_{n}\left(z_{n}\right)\right)+K A_{n} \stackrel{n}{\longrightarrow} 0
$$

so that, by local equicontinuity

$$
\lim _{n}\left|f_{n}\left(z_{n}\right)-f_{n}\left(y_{n}\right)\right|=0
$$

which in the end implies

$$
\lim _{n} f_{n}\left(y_{n}\right)=f\left(x_{0}\right)
$$

as desired.

Next proposition put in relation local global and uniform $F_{n}$-convergence.

2.3. Proposition. Assume $\lim _{n}\left(\mathcal{M}_{n}, d_{n}, F_{n}\right)=(\mathcal{M}, d)$, let $f_{n}:\left(\mathcal{M}_{n}, d_{n}\right) \rightarrow \mathbb{R}$ be continuous. If $f_{n}$ locally uniformly $F_{n}$-converges to some function $f:(\mathcal{M}, d) \rightarrow \mathbb{R}$ then

$$
\lim _{n} \sup _{x \in F_{n}^{-1}(\mathbb{K})}\left|f_{n}(x)-f\left(F_{n}(x)\right)\right|=0 \quad \text { for any compact subset } \mathbb{K} \text { of } \mathcal{M} \text {. }
$$

Conversely, if (38) holds and $f$ is continuous then $f_{n}$ locally uniformly $F_{n}$-converges to $f$.

Proof: First, assume $f_{n}$ locally uniformly convergent to $f$. If 38 were not true, there should be a compact subset of $\mathbb{K} \subset \mathcal{M}, \delta>0$, and a (sub)sequence $y_{n} \in F_{n}^{-1}(\mathbb{K})$ with

$$
\left|f_{n}\left(y_{n}\right)-f\left(F_{n}\left(y_{n}\right)\right)\right|>\delta
$$

Since $F_{n}\left(y_{n}\right)$ converges, up to subsequences, to some $x \in \mathbb{K}$, we get by local uniform convergence $\lim _{n} f_{n}\left(y_{n}\right)=f(x)$, being $f$ continuous by Proposition 2.1, we also have $\lim _{n} f\left(F_{n}\left(y_{n}\right)\right)=f(x)$. These two limit relations are in contrast with 39].

Conversely, if (38) holds and a (sub)sequence $F_{n}\left(y_{n}\right) \in \mathcal{M}_{n}$ converges to $x \in \mathcal{M}$, then $y_{n} \in F_{n}^{-1}(\mathbb{K})$, for some compact subset of $\mathcal{M}$ and

$$
\left|f_{n}\left(y_{n}\right)-f\left(F_{n}\left(y_{n}\right)\right)\right| \longrightarrow 0
$$

the fact that $f$ is continuous by assumption implies $\lim _{n} f\left(F_{n}\left(y_{n}\right)\right)=f(x)$, this last relation, combined with (40), yields

$$
\lim _{n} f_{n}\left(y_{n}\right)=f(x)
$$




\section{Homogenization in the MAXimal FREe ABELIAN COVER}

Write

$$
\begin{aligned}
H_{\varepsilon}(x, p): & =\widetilde{H}\left(x, \frac{1}{\varepsilon} p\right) . \\
L_{\varepsilon}(x, v): & =\max _{p \in T_{x}^{*} M}\left\{p \cdot v-H_{\varepsilon}(x, p)\right\} \\
& =\max _{p \in T_{x}^{*} M}\left\{\frac{p}{\varepsilon} \cdot(v \varepsilon)-\widetilde{H}\left(x, \frac{p}{\varepsilon}\right)\right\} \\
& =L(x, \varepsilon v) .
\end{aligned}
$$

The solution to the problem (18)-(19) is given by the Lax-Oleinik formula

$$
\begin{aligned}
v^{\varepsilon}(x, t) & =\inf \left\{f_{\varepsilon}(\gamma(0))+\int_{0}^{t} L_{\varepsilon}(\gamma, \dot{\gamma}) d t \mid \gamma \in C^{1}([0, t], \widetilde{M}), \gamma(t)=x\right\}, \\
& =\inf _{\gamma(t)=x}\left\{f_{\varepsilon}(\gamma(0))+\int_{0}^{t} L(\gamma, \varepsilon \dot{\gamma})\right\} .
\end{aligned}
$$

Write $\eta:\left[0, \frac{t}{\varepsilon}\right] \rightarrow \widetilde{M}, \eta(s):=\gamma(\varepsilon s)$. Then

$$
\begin{aligned}
\int_{0}^{t} L(\gamma, \varepsilon \dot{\gamma}) d t & =\int_{0}^{\frac{t}{\varepsilon}} L(\eta(s), \dot{\eta}(s)) \varepsilon d s \\
v^{\varepsilon}(x, T) & =\inf \left\{f_{\varepsilon}(\eta(0))+\varepsilon \int_{0}^{\frac{t}{\varepsilon}} L(\eta, \dot{\eta}) d s \mid \eta \in C^{1}\left(\left[0, \frac{t}{\varepsilon}\right], \widetilde{M}\right), \eta\left(\frac{t}{\varepsilon}\right)=x\right\}, \\
& =\inf _{y \in \widetilde{M}}\left\{f_{\varepsilon}(y)+\varepsilon \tilde{\phi}\left(y, x, \frac{t}{\varepsilon}\right)\right\}
\end{aligned}
$$

where

$$
\tilde{\phi}(y, x, t):=\inf \left\{\int_{0}^{t} L(\eta, \dot{\eta}) d s \mid \eta \in C^{1}([0, S], \widetilde{M}), \eta(0)=y, \eta(t)=x\right\} .
$$

The solution to the limit problem $(20)-210$ is

$$
u(h, t)=\inf _{q \in H_{1}(M, \mathbb{R})}\left\{f(q)+t \beta\left(\frac{h-q}{t}\right)\right\},
$$

where $\beta$ is Mather's minimal action functional (23). Optimal elements for $u(h, t)$ do exists because $f$ is continuous by Proposition 2.1 and with linear growth, $\beta$ superlinear.

The proof is just to show that formula (42) converges to formula (43), using Mather's proposition 3.1 below on the uniform convergence of mean minimal actions to the beta function. See [10, Corollary on page 181], we have just slightly changed notations: 
3.1. Proposition. For every $A>0, \delta>0$ there is $T_{0}>0$ such that if $\quad x, y \in \widetilde{M}, \quad T \geq T_{0}, \quad\left\|\frac{G(y)-G(x)}{T}\right\| \leq A, \quad$ then

$$
\left|\frac{1}{T} \tilde{\phi}(x, y, T)-\beta\left(\frac{G(y)-G(x)}{T}\right)\right|<\delta .
$$

We recall that the element $G(y)-G(x)$ of $H_{1}(M, \mathbb{R})$ is characterized by the relation

$$
\left\langle c_{i}, G(y)-G(x)\right\rangle=\oint_{x}^{y} \widetilde{\omega}_{i}
$$

for any $i=1, \cdots, k$, where $c_{1}, \cdots, c_{k}$ is a basis of $H_{1}(M, \mathbb{R}), \omega_{i}$ are representatives in $c_{i}$ and $\widetilde{\omega}_{i}$ are the lifts of $\omega_{i}$ to $\widetilde{M}$, see 17 . We deduce from Proposition 3.1

3.2. Proposition. Let $x_{\varepsilon}$, $y_{\varepsilon}$ be in $\widetilde{M}$, for any $\varepsilon>0$, and $h, q$ in $H_{1}(M, \mathbb{R})$. Let $t_{\varepsilon}$ be a sequence of positive times converging to $t_{0}>0$. If $\lim _{\varepsilon} F_{\varepsilon}\left(x_{\varepsilon}\right)=h, \lim _{\varepsilon} F_{\varepsilon}\left(y_{\varepsilon}\right)=q$ then

$$
\lim _{\varepsilon} \varepsilon \tilde{\phi}\left(y_{\varepsilon}, x_{\varepsilon}, \frac{t_{\varepsilon}}{\varepsilon}\right)=t_{0} \beta\left(\frac{h-q}{t_{0}}\right) .
$$

Proof: Let $a, b$ be constants estimating from below and above, respectively, $t_{\varepsilon}$, at least for $\varepsilon$ suitably small. We take $A, \delta, T_{0}$ as in Proposition 3.1. For $\varepsilon$ small we have

$$
\left\|\frac{\varepsilon\left(G\left(x_{\varepsilon}\right)-G\left(y_{\varepsilon}\right)\right)}{a}\right\| \leq A \quad \text { and } \quad \frac{a}{\varepsilon}>T_{0} .
$$

We derive in force of Proposition 3.1,

$$
\left|\varepsilon \tilde{\phi}\left(y_{\varepsilon}, x_{\varepsilon}, \frac{t_{\varepsilon}}{\varepsilon}\right)-t_{\varepsilon} \beta\left(\frac{F_{\varepsilon}\left(x_{\varepsilon}\right)-F_{\varepsilon}\left(y_{\varepsilon}\right)}{t_{\varepsilon}}\right)\right|<b \delta \quad \text { for } \varepsilon \text { small. }
$$

Therefore

$$
\lim _{\varepsilon}\left|\varepsilon \tilde{\phi}\left(y_{\varepsilon}, x_{\varepsilon}, \frac{t_{\varepsilon}}{\varepsilon}\right)-t_{\varepsilon} \beta\left(\frac{F_{\varepsilon}\left(x_{\varepsilon}\right)-F_{\varepsilon}\left(y_{\varepsilon}\right)}{t_{\varepsilon}}\right)\right|=0,
$$

and the assertion follows being $\beta(\cdot)$ continuous.

3.3. Lemma. Given a compact subset $\mathbb{K}$ in $H_{1}(M, \mathbb{R})$, and a compact interval $\left.I \subset\right] 0,+\infty[$, there is a positive constant $C$ such that

$$
d_{\varepsilon}(x, y) \leq C
$$

for $\varepsilon>0$ suitably small, any $x \in F_{\varepsilon}^{-1}(\mathbb{K}), t \in I$ and $y \in \widetilde{M}$ realizing the minimum in the formula yielding $v^{\varepsilon}(x, t)$.

Proof: By linear growth assumption on $f$ we find

$$
f(h) \geq-A\|h\|-B
$$


for any $h \in H_{1}(M, \mathbb{R})$ and suitable positive constants $A$ and $B$, by applying uniform convergence of $f_{\varepsilon}$ to $f$ we infer

$$
f_{\varepsilon}(x) \geq-A \varepsilon\|G(x)\|-(B+1) \quad x \in \widetilde{M}
$$

for $\varepsilon$ suitably small and, in addition, we find a constant $Q$ with

$$
f_{\varepsilon}(x) \leq Q \quad \text { for } \varepsilon \text { small, } x \in F_{\varepsilon}^{-1}(\mathbb{K}) .
$$

Since by condition (a) in the definition of spaces convergence $\|G(x)\|$ and $d(x, y)$ are infinite of the same order, as $d\left(x, x_{0}\right) \rightarrow+\infty$, for any fixed $x_{0}$, we deduce from (45), up to adjusting constants $A, B$

$$
f_{\varepsilon}(x) \geq-A d_{\varepsilon}\left(x, x_{0}\right)-(B+1) \quad x \in \widetilde{M} .
$$

By superlinearity of $L$ we are able to find, for any $M>0$, a positive $N$ such that, taken a pair $x, y$ of elements of $\widetilde{M}$ and any curve $\gamma$ linking them in $[0, t]$, one has

$$
\int_{0}^{t} L(\gamma, \varepsilon \dot{\gamma}) d t \geq M d_{\varepsilon}(x, y)-N t
$$

By 46

$$
v^{\varepsilon}\left(x_{0}, t\right) \leq Q+t \min _{y \in M} L(y, 0) \quad \text { for } \varepsilon \text { small, } x_{0} \in F_{\varepsilon}^{-1}(\mathbb{K}) .
$$

By combining this last inequality (47), 48), and taking into account the very definition of $v^{\varepsilon}$, we get the assertion.

\section{Proof of theorem 1.2:}

Let $\left(h_{0}, t_{0}\right)$ be in $\left.H_{1}(M, \mathbb{R}) \times\right] 0,+\infty\left[\right.$. Let $\left(x_{\varepsilon}, t_{\varepsilon}\right)$ be a (sub)sequence in $\left.\widetilde{M} \times\right] 0,+\infty[$ with $\lim _{\varepsilon} F_{\varepsilon}\left(x_{\varepsilon}\right)=h_{0}, \lim _{\varepsilon} t_{\varepsilon}=t_{0}$, our task is to show

$$
\lim _{\varepsilon} v^{\varepsilon}\left(x_{\varepsilon}, t_{\varepsilon}\right)=u\left(h_{0}, t_{0}\right) \text {. }
$$

Assume a subsequence $v^{\varepsilon_{k}}\left(x_{\varepsilon_{k}}, t_{\varepsilon_{k}}\right)$ to have limit and consider $y_{\varepsilon_{k}}$ optimal for $v^{\varepsilon_{k}}\left(x_{\varepsilon_{k}}, t_{\varepsilon_{k}}\right)$, then, according to Lemma $3.3, d_{\varepsilon_{k}}\left(x_{\varepsilon_{k}}, y_{\varepsilon_{k}}\right)<C$ for $\varepsilon_{k}$ small, which implies that $\| F_{\varepsilon}\left(x_{\varepsilon_{k}}\right)-$ $F_{\varepsilon}\left(y_{\varepsilon_{k}}\right) \|$, and consequently $\| h_{0}-F_{\varepsilon}\left(y_{\varepsilon_{k}} \|\right.$ are bounded. We deduce that $F_{\varepsilon_{k}}\left(y_{\varepsilon_{k}}\right)$ converges, up to subsequences, to some element $q_{0} \in H_{1}(M, \mathbb{R})$. We therefore get in force of Proposition 3.2

$$
\lim _{\varepsilon_{k}} \varepsilon_{k} \tilde{\phi}\left(y_{\varepsilon_{k}}, x_{\varepsilon_{k}}, \frac{t_{\varepsilon_{k}}}{\varepsilon_{k}}\right)=t_{0} \beta\left(\frac{h_{0}-q_{0}}{t_{0}}\right) .
$$

Exploiting the uniform $F_{\varepsilon}$-convergence of $f_{\varepsilon}$ to $f$, we further derive

$$
\lim _{\varepsilon_{k}} f\left(y_{\varepsilon_{k}}\right)+\varepsilon_{k} \tilde{\phi}\left(y_{\varepsilon_{k}}, x_{\varepsilon_{k}}, \frac{t_{\varepsilon_{k}}}{\varepsilon_{k}}\right)=f\left(q_{0}\right)+t_{0} \beta\left(\frac{h_{0}-q_{0}}{t_{0}}\right) \geq u\left(h_{0}, t_{0}\right)
$$

and consequently

$$
\liminf _{\varepsilon} v^{\varepsilon}\left(x_{\varepsilon}, t_{\varepsilon}\right) \geq u\left(h_{0}, t_{0}\right)
$$


We denote by $\bar{q}$ an optimal element for $u\left(h_{0}, t_{0}\right)$, there is a sequence $y_{\varepsilon}$ in $\widetilde{M}$ with $\lim _{\varepsilon} F_{\varepsilon}\left(y_{\varepsilon}\right)=\bar{q}$, therefore, again by Proposition 3.2 and convergence of $f_{\varepsilon}$ to $f$ we get

$$
\lim \sup v^{\varepsilon}\left(x_{\varepsilon}, t_{\varepsilon}\right) \leq \lim _{\varepsilon} f_{\varepsilon}\left(y_{\varepsilon}\right)+\varepsilon \tilde{\phi}\left(y_{\varepsilon}, x_{\varepsilon}, \frac{t_{\varepsilon}}{\varepsilon}\right)=f(\bar{q})+t_{0} \beta\left(\frac{h_{0}-\bar{q}}{t_{0}}\right)=u\left(q_{0}, t_{0}\right),
$$

this limit relation together with $(50)$ gives $(49)$.

\section{SUBCOVERS}

\section{Proof of Theorem 1.4;}

Consider the lifts to $\widetilde{M}$ of the solutions $v^{\varepsilon}$ and the initial conditions $f_{\varepsilon}$, as well as the the lift to $H_{1}(M, \mathbb{R})$ of $f$ :

$$
\tilde{v}^{\varepsilon}(x, t):=v^{\varepsilon}(\pi(x), t), \quad \tilde{f}_{\varepsilon}(x)=f_{\varepsilon}(\pi(x)), \quad \tilde{f}(h)=f(\mathfrak{f}(h)) .
$$

Due to the fact that $\widehat{H}$ is the lift of an Hamiltonian $H$ defined in $T^{*} M$ and the differential structures of $\widetilde{M}, \widehat{M}$ are the same, $\tilde{v}^{\varepsilon}(x, t)$ is solution to 18$)$ with initial datum $\tilde{f}_{\varepsilon}(x)$. Given $\varepsilon>0$ and $x_{0} \in \widetilde{M}$, we have

$$
\left|\tilde{f}_{\varepsilon}\left(x_{0}\right)-\tilde{f}\left(F_{\varepsilon}\left(x_{0}\right)\right)\right|=\mid f_{\varepsilon}\left(\pi\left(x_{0}\right)\right)-f\left(\mathfrak{f}\left(F_{\varepsilon}\left(x_{0}\right)\right)|=| f_{\varepsilon}\left(\pi\left(x_{0}\right)\right)-f\left(\widehat{F}_{\varepsilon}\left(\pi\left(x_{0}\right)\right)\right) \mid,\right.
$$

in addition if $\lim _{\varepsilon} F_{\varepsilon}\left(x_{\varepsilon}\right)=h_{0}$ for some sequence $x_{\varepsilon}$ in $\widetilde{M}, h_{0} \in H_{1}(M, \mathbb{R})$, then

$$
\lim _{\varepsilon} \widehat{F}\left(\pi\left(x_{\varepsilon}\right)\right)=\lim _{\varepsilon} \mathfrak{f} F_{\varepsilon}\left(x_{\varepsilon}\right)=\mathfrak{f}\left(h_{0}\right)
$$

then, by the uniform $\widehat{F}_{\varepsilon}$-convergence of $f_{\varepsilon}$ to $f$ as $\varepsilon \rightarrow 0$

$$
\lim _{\varepsilon} \tilde{f}_{\varepsilon}\left(x_{\varepsilon}\right)=\lim _{\varepsilon} f_{\varepsilon}\left(\pi\left(x_{\varepsilon}\right)\right)=f\left(\mathfrak{f}\left(h_{0}\right)\right)=\tilde{f}\left(h_{0}\right)
$$

this limit relation together (51) shows the uniform $F_{\varepsilon}$-convergence of $\tilde{f}_{\varepsilon}$ to $\tilde{f}$. By Theorem 1.2 we therefore get that $\tilde{v}^{\varepsilon}(x, t)$ locally uniformly $F_{\varepsilon}$-converges to

$$
\tilde{u}(q, t):=\inf _{h \in \mathbb{H}_{1}(M, \mathbb{R})}\left\{\tilde{f}(h)+t \beta\left(\frac{q-h}{t}\right)\right\} \quad(q, t) \in H_{1}(M, \mathbb{R}) \times[0,+\infty[.
$$

If $z \in \operatorname{ker} \mathfrak{f}$ then

$$
\begin{aligned}
\tilde{u}(q+z, t) & =\inf _{h \in \mathbb{H}_{1}(M, \mathbb{R})}\left\{\tilde{f}(h)+t \beta\left(\frac{q+z-h}{t}\right)\right\} \\
& =\inf _{h \in \mathbb{H}_{1}(M, \mathbb{R})}\left\{\tilde{f}(h-z)+t \beta\left(\frac{q+z-h}{t}\right)\right\}=\tilde{u}(q, t) .
\end{aligned}
$$


From this we recognize that $\tilde{u}$ is the lift to $H_{1}(M, \mathbb{R})$ of a function $u$ defined in $\operatorname{im} \mathfrak{f} \times$ $\left[0,+\infty\left[\right.\right.$. Using $(25)$, we have for any $q_{0} \in \operatorname{im} \mathfrak{f}, h_{0} \in H_{1}(M, \mathbb{R})$ with $\mathfrak{f}\left(h_{0}\right)=q_{0}$

$$
\begin{aligned}
u\left(q_{0}, t\right) & =\inf _{z \in \operatorname{ker} \mathfrak{f}} \tilde{u}\left(h_{0}+z, t\right)=\inf _{h \in H_{1}(M, \mathbb{R})}\left\{\tilde{f}(h)+\inf _{z \in \operatorname{ker} \mathfrak{f}} t \beta\left(\frac{h_{0}+z-h}{t}\right)\right\} \\
& =\inf _{q \in \operatorname{imf} \mathfrak{f}}\left\{f(q)+t \widehat{\beta}\left(\frac{q_{0}-q}{t}\right)\right\},
\end{aligned}
$$

so that $u$ is the solution to the limit problem (30)-(31). It is left to investigate the convergence of $v^{\varepsilon}$ to $u$. We consider $\left.\left(q_{0}, t_{0}\right) \in \mathrm{im} \mathfrak{f} \times\right] 0,+\infty\left[\right.$, a (sub)sequence $\left(y_{\varepsilon}, t_{\varepsilon}\right) \in$ $\widehat{M} \times] 0,+\infty\left[\right.$ with $\lim _{\varepsilon} \widehat{F}_{\varepsilon}\left(y_{\varepsilon}\right)=q_{0}, \lim _{\varepsilon} t_{\varepsilon}=t_{0}$; then there is a compact subset $\mathbb{K}$ of $\operatorname{im} \mathfrak{f}$ with

$$
\mathfrak{f} F_{\varepsilon}\left(x_{\varepsilon}\right) \in \mathbb{K} \quad \text { for } \varepsilon \text { small, } x_{\varepsilon} \in \pi^{-1}\left(y_{\varepsilon}\right) .
$$

We can select $\mathbb{K}_{0} \subset \mathfrak{f}^{-1}(\mathbb{K}), \mathbb{K}_{0}$ compact in $H_{1}(M, \mathbb{R})$, with $\mathfrak{f}\left(\mathbb{K}_{0}\right)=\mathbb{K}$, in addition, taking into account that $H_{1}(M, \mathbb{Z}) \cap \operatorname{ker} \mathfrak{f}$ is $B$-dense in ker $\mathfrak{f}$ and (24), we infer that there is a compact enlargement $\mathbb{K}_{1}$ of $\mathbb{K}_{0}$ such that

$$
\left\{x_{\varepsilon} \in \pi^{-1}\left(y_{\varepsilon}\right) \mid F_{\varepsilon}\left(x_{\varepsilon}\right) \in \mathbb{K}_{1}\right\} \neq \emptyset \text { for } \varepsilon \text { small. }
$$

Choosing $F_{\varepsilon}\left(x_{\varepsilon}\right)$, for $x_{\varepsilon}$ in the above set, we build up a sequence converging, up to a subsequence, to some $h_{0} \in H_{1}(M, \mathbb{R})$ with $\mathfrak{f}\left(h_{0}\right)=q_{0}$, then by local uniform $F_{\varepsilon}$-convergence of $\tilde{v}^{\varepsilon}$ to $\tilde{u}$ we get

$$
\lim _{\varepsilon} v^{\varepsilon}\left(y_{\varepsilon}, t_{\varepsilon}\right)=\lim _{\varepsilon} \tilde{v}^{\varepsilon}\left(x_{\varepsilon}, t_{\varepsilon}\right)=\tilde{u}\left(h_{0}, t_{0}\right)=u\left(q_{0}, t_{0}\right) .
$$

This fact shows the sought local uniform $\widehat{F}_{\varepsilon}$-convergence of $v^{\varepsilon}$ to $u$ and concludes the proof.

\section{REFERENCES}

[1] Gonzalo Contreras and Renato Iturriaga, Global Minimizers of Autonomous Lagrangians, $22^{\circ}$ Coloquio Bras. Mat., IMPA, Rio de Janeiro, 1999.

[2] Gonzalo Contreras, Renato Iturriaga, Gabriel P. Paternain, and Miguel Paternain, Lagrangian graphs, minimizing measures and Mañé's critical values, Geom. Funct. Anal. 8 (1998), no. 5, 788-809.

[3] Michael G. Crandall and Pierre-Louis Lions, On existence and uniqueness of solutions of HamiltonJacobi equations, Nonlinear Anal. 10 (1986), no. 4, 353-370.

[4] Andrea Davini and Maxime Zavidovique, On the (non) existence of viscosity solutions of multi-time Hamilton-Jacobi equations, Preprint 2013.

[5] Albert Fathi, Weak KAM Theorem in Lagrangian Dynamics, To appear.

[6] _ Théorème KAM faible et théorie de Mather sur les systèmes lagrangiens, C. R. Acad. Sci. Paris Sér. I Math. 324 (1997), no. 9, 1043-1046.

[7] Hitoshi Ishii, Remarks on existence of viscosity solutions of Hamilton-Jacobi equations, Bull. Fac. Sci. Engrg. Chuo Univ. 26 (1983), 5-24.

[8] P.-L. Lions, G. Papanicolau, and S. R. S. Varadhan, Homogenization of Hamilton-Jacobi equations, preprint, unpublished, 1987. 
[9] Ricardo Mañé, Lagrangian flows: the dynamics of globally minimizing orbits, International Conference on Dynamical Systems (Montevideo, 1995), Longman, Harlow, 1996, Reprinted in Bol. Soc. Brasil. Mat. (N.S.) 28 (1997), no. 2, 141-153., pp. 120-131.

[10] John N. Mather, Action minimizing invariant measures for positive definite Lagrangian systems, Math. Z. 207 (1991), no. 2, 169-207.

Cimat, A.P. 402, 36.000, Guanajuato. GTO, MÉxico.

E-mail address: gonzalo@cimat.mx

CimAt, A.P. 402, 36.000, Guanajuato. GTO, MÉxico.

E-mail address: renato@cimat.mx

Dipartimento di Matematica, Università degli Studi di Roma "La Sapienza", Piazzale Aldo Moro 2, 00185 Roma, Italy.

E-mail address: siconolf@mat.uniroma1.it 